\title{
Crohn disease-like enterocolitis remission after empagliflozin treatment in a child with glycogen storage disease type lb: a case report
}

\author{
Alessandro Rossi ${ }^{1,2^{*}}$ (D), Erasmo Miele ${ }^{1}$, Simona Fecarotta ${ }^{1}$, Maria Veiga-da-Cunha ${ }^{3}$, Massimo Martinelli ${ }^{1}$, \\ Carmine Mollica ${ }^{4}$, Maria D'Armiento ${ }^{5}$, Enza Mozzillo', Pietro Strisciuglio', Terry G. J. Derks², Annamaria Staiano ${ }^{1}$ and \\ Giancarlo Parenti ${ }^{1,6}$
}

\begin{abstract}
Background: Besides major clinical/biochemical features, neutropenia and inflammatory bowel disease (IBD) constitute common complications of Glycogen storage disease type lb (GSD Ib). However, their management is still challenging. Although previous reports have shown benefit of empagliflozin administration on neutropenia, no follow-up data on bowel (macro/microscopic) morphology are available. We herein present for the first time longitudinal assessment of bowel morphology in a GSD Ib child suffering from Crohn disease-like enterocolitis treated with empagliflozin.
\end{abstract}

Case presentation: A 14-year-old boy with GSD Ib and severe IBD was (off-label) treated with empagliflozin (20 mg/day) after informed oral and written consent was obtained from the patient's parents. No adverse events were noted. Clinical symptoms and stool frequency improved within the first week of treatment. Pediatric Crohn disease activity index (PCDAl) normalised within the first month of treatment. Abdomen magnetic resonance imaging (MRI) performed 3 months after treatment initiation showed dramatic decrease in disease activity and length. Similar findings were reported on histology at 5.5 months. At 7.5 months hemoglobin levels normalised and fecal calprotectin almost normalised. Improved neutrophil count, metabolic control and quality of life were also noted. G-CSF dose was decreased by 33\% and the patient was partly weaned from tube feeding.

Conclusions: This is the first report presenting extensive gastrointestinal morphology follow-up in a GSD Ib patient receiving empagliflozin. The present case suggests that empagliflozin can be safe and effective in inducing IBD remission in GSD Ib patients and can even postpone surgery. Future studies are required to confirm its effect over time and assess its benefit in various disease stages. The development of an international collaborating networks for systematic data collection is worthy.

Keywords: Glycogen storage disease type Ib, Inflammatory bowel disease, Neutropenia, 1,5-anhydroglucitol, Empagliflozin, Continuous glucose monitoring

\footnotetext{
*Correspondence: alessandro.rossi@unina.it; a.rossi@umcg.nl

'Department of Translational Medical Sciences, Section of Pediatrics,

University of Naples "Federico II", Naples, Italy

${ }^{2}$ Section of Metabolic Diseases, Beatrix Children's Hospital, University Medical

Center Groningen, University of Groningen, P.O. Box 30.001, 9700 RB

Groningen, The Netherlands

Full list of author information is available at the end of the article
}

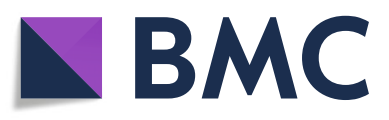

(c) The Author(s). 2021 Open Access This article is licensed under a Creative Commons Attribution 4.0 International License, which permits use, sharing, adaptation, distribution and reproduction in any medium or format, as long as you give appropriate credit to the original author(s) and the source, provide a link to the Creative Commons licence, and indicate if changes were made. The images or other third party material in this article are included in the article's Creative Commons licence, unless indicated otherwise in a credit line to the material. If material is not included in the article's Creative Commons licence and your intended use is not permitted by statutory regulation or exceeds the permitted use, you will need to obtain permission directly from the copyright holder. To view a copy of this licence, visit http://creativecommons.org/licenses/by/4.0/ The Creative Commons Public Domain Dedication waiver (http://creativecommons.org/publicdomain/zero/1.0/) applies to the data made available in this article, unless otherwise stated in a credit line to the data. 


\section{Background}

Glycogen storage disease type Ib (GSD Ib, MIM\#232220) is an inherited disorder of carbohydrate metabolism due to microsomal glucose-6-phosphate transporter (G6PT) deficiency (SLC37A4 gene). The ubiquitously expressed G6PT transports glucose 6-phosphate (G6P) from cytosol to endoplasmic reticulum (ER) where it is oxidized to glucose to ensure glucose homeostasis. G6PT defect results into both glycogenolysis and gluconeogenesis defect [1]. Major clinical features of GSD Ib include fasting hypoglycaemia, hyperlactatemia, hyperuricemia, hyperlipidaemia, hepatomegaly, growth retardation, renal disease [2]. Additionally, GSD Ib patients show neutropenia/neutrophil dysfunction [3] and increased risk of inflammatory bowel disease (IBD) (i.e., Crohn disease-like enterocolitis) [4], and autoimmune disorders $[5,6]$.

Despite the progress in the (medical and dietary) treatment of GSD Ib over the past years, such immunological complications still heavily impact on patients' prognosis and quality of life. While evidence regarding the pathogenesis of neutropenia/neutrophil dysfunction and autoimmune disorders has accumulated [7-9], the pathomechanism of IBD in GSD Ib is still unclear; the disturbed immune response may play a role in its pathogenesis [4]. Granulocyte-colony stimulating factor (GCSF) for neutropenia and conventional drugs for IBD and autoimmune disorders still constitute the current treatment options for most GSD Ib patients. For IBD, conventional treatments are sometimes ineffective and/ or associated with side effects and patients might eventually need surgery [1]. Notably, improved prevention/ treatment of IBD in GSD Ib ranked as a top priority for research in the international priority setting partnership for liver glycogen storage diseases [10].

Recent evidence has shown a major role for plasma 1,5anhydroglucitol (1,5AG) in causing neutropenia/neutrophil dysfunction in GSD Ib. 1,5AG enters neutrophils where it is phosphorylated to 1,5-anhydroglucitol-6-phosphate (1, 5AG6P). 1,5AG6P is transported by G6PT into the ER, where it is physiologically dephosphorylated. G6PT defect results into cytosolic toxic 1,5AG6P accumulation thus affecting neutrophils survival and function [11].

Empagliflozin is a sodium glucose co-transporter 2 (SGLT2) inhibitor approved for the treatment of type 2 diabetes which reduces renal 1,5AG resorption by increasing urinary glucose excretion. Notably, empagliflozin administration decreased 1,5AG (plasma) and 1, 5AG6P (neutrophils) concentrations in GSD Ib mice [11]. Two recent reports have shown same effect in GSD Ib patients with improved neutrophil count/ function. Possible benefit on gastrointestinal symptoms have also been reported [12, 13]. However, no follow-up data on bowel (macro/microscopic) morphology are available.
We herein present for the first time longitudinal assessment of bowel morphology in a GSD Ib child suffering from Crohn disease-like enterocolitis treated with empagliflozin.

\section{Case presentation \\ Methods \\ Study design}

Empagliflozin is a SGLT2-inhibitor registered and marketed for type 2 diabetes in adults. Its most common adverse effects include low blood pressure and urogenital infections [14]. In the case herein described informed oral and written consent for the off-label treatment with empagliflozin was obtained from the patient and patient's parents after discussing potential benefits and adverse effects of such treatment. Baseline data were collected 1 (day -1) or 2 (day -2) days before starting the treatment during in-hospital admission under medical supervision (day 0). Vital parameters were checked every $2 \mathrm{~h}$ within the first $12 \mathrm{~h}$ after treatment initiation and subsequently every $8 \mathrm{~h}$. The patient was discharged on day +5 . Regular assessments of his GSD Ib and related conditions were performed at the outpatient clinic every 1 week within the first 2 months of treatment; subsequent evaluations were performed based on the patient's conditions and medical advice. Blood samples were collected at the maximum distance after last CGSF administration (day -2 to day 30: $48 \mathrm{~h}$; day 37 to day 51: $72 \mathrm{~h}$; day 64 to day $71: 96 \mathrm{~h}$; day 78 to day 115 : $72 \mathrm{~h})$. Physical examination included: weight, height and body mass index, signs/symptoms of infections, abdominal pain, mouth ulcers and perianal lesions. Adverse events were also recorded. For all results, the specific day of collection (i.e., day before/after starting the treatment) is reported in the main text, tables or figures.

\section{Gastrointestinal assessment}

An expert endoscopist performed the colonoscopy. During colonoscopy, 4 biopsies were taken from each colonic segment and from the terminal ileum, if entered. The histologic features were assessed by an experienced IBD pathologist, who was blinded to the endoscopic features and clinical history of the patients. Magnetic Resonance Imaging (MRI) was performed by an experienced IBD radiologist (who was blinded to the morphological features and clinical history of the patient) using a highfield (3.0-Tesla) scanner (Trio, Siemens) using a body coil with four channels; the following sequences were acquired: T2-weighted HASTE triggered on the axial plane (TR/TE 2000/91 ms; thickness $6 \mathrm{~mm}$; flip angle 150; matrix: $256 \times 157$; acquisition time: $64 \mathrm{~s}$ ), T2-weighted HASTE triggered on the coronal plane (TR/TE 2000/92 ms; thickness $4 \mathrm{~mm}$; flip angle 121 ; matrix $320 \times 256$; acquisition time $80 \mathrm{~s}$ ) with and without fat saturation, T1- 
weighted in-phase on the axial plane (TR/TE 1500/2.3 ms; thickness $6 \mathrm{~mm}$; flip angle 20; matrix $256 \times 154$; acquisition time $50 \mathrm{~s}$ ), T1- weighted out-of-phase on the axial plane (TR/TE 1500/1.37 ms; thickness $3.5 \mathrm{~mm}$; flip angle 20; matrix $256 \times 160$; acquisition time $58 \mathrm{~s}$ ) before and after intravenous injection of paramagnetic contrast (gadopentetate dimeglumine, Magnevist, Bayer HealthCare Pharmaceuticals). Disease activity was assessed using the pediatric Crohn disease activity index (PCDAI) [15]. Stool consistency was assessed with the Bristol stool chart.

\section{Biochemical tests}

Fecal calprotectin was assessed through ELISA assay. Plasma 1,5AG and granulocytes 1,5AG6P were assessed as previously described [12]. Blood (glucose, lactate, cholesterol, triglycerides (TG), uric acid, AST, ALT, albumin, complete blood count, absolute neutrophil count (ANC), C-reactive protein (CRP), erythrocyte sedimentation rate (ESR) within the first hour, creatinine, blood urea nitrogen) and urine tests (creatinine, (24 h-proteins, $24 \mathrm{~h}$-glucose, urinalysis) were performed by using assays with commercially available kits.

\section{Glucose monitoring}

Besides capillary glucose measurements, flash glucose monitoring (FGM) was performed through an intermittent scanning FGM device (Freestyle Libre2) during the following time frames: 1) baseline to day $+10 ; 2)+3$ months; 3$)+5.5$ months; 4$)+7.5$ months; 5$)+8$ months. Low-glucose threshold was set at $3.3 \mathrm{mmol} / \mathrm{L}$. In case of glucose concentrations below threshold, capillary glucose was also checked. Hypoglycemia was defined as capillary glucose $<3.3 \mathrm{mmol} / \mathrm{L}$. Due to possible interference of daily life activities, physical activity and the risk of temporary sensor disconnection, both 24-h and nighttime (1 a.m. to 5 a.m.) FGM data were analyzed for each time frame by using descriptive statistics. Only days with $>15$ time points available were considered for the analysis. Time below range (TBR), time in range (TIR) and time above range (TAR) were defined according to current consensus glucose monitoring recommendations [16].

\section{Quality of life (QoL)}

Health-related QoL was assessed at baseline and on day +232 through the Italian version of the Short Form Health Survey (SF-36) questionnaire which has been previously used for GSDI patients. The SF-36 questionnaire consists of 36 items combined into eight scaled scores. The raw score is transformed into a $0-100$ scale to generate a summary measure, with higher scores indicating better QoL [17].

\section{Case presentation}

A 14-year-old boy was diagnosed with GSD Ib at age 7 months due to fasting hypoketotic hypoglycemia with high lactate, enlarged liver and neutropenia. The molecular diagnosis of the SLC37A4 gene showed homozygosity for the mutation c.742C > T (p.Gln248X). A diet based on frequent meals and nocturnal gastric dripfeeding was started and the patient was included in a follow-up program at Section of Pediatrics, University of Naples "Federico II". Several unsuccessful attempts with uncooked cornstarch were made in order to extend his fasting time (all associated with diarrhea, abdominal pain, vomiting). After such attempts, the patient developed avoidant/restrictive food intake disorder. Starting from age 6 years, 24-h gastric drip feeding (GDF) was required. Due to neutropenia, he was also started with i.m. G-CSF.

During the follow-up several complications appeared. At 9 years of age juvenile idiopathic arthritis was diagnosed following the development of arthralgia and arthritis in the right knee and hip. From that age he also experienced recurrent aphthous stomatitis. Since Naproxen $(15 \mathrm{mg} / \mathrm{Kg} /$ day $)$ administration showed no benefit, i.m. methotrexate $\left(15 \mathrm{mg} / \mathrm{m}^{2}\right.$ every $1-3$ weeks $)$ was started. At age 13 limitation of range of motion and arthritis in the left knee required intra-articular injection of triamcinolone acetonide. At age 14 hyperuricemia was detected, requiring allopurinol administration $(200 \mathrm{mg}$ twice per day). Kidney function was regularly assessed and found normal. Plasma cholesterol (range 1.4-2.5 $\mathrm{mmol} / \mathrm{L}$ ) and TG (range $0.4-1.3 \mathrm{mmol} / \mathrm{L}$ ) concentrations were constantly decreased.

Since 10 years of age, he suffered from Crohn-like IBD (PCDAI:75 at the diagnosis). Chronic anemia was also detected requiring several (partly beneficial) intravenous iron infusions (Hb 7.6-10.5 g/dL). Despite methotrexate $\left(15 \mathrm{mg} / \mathrm{m}^{2}\right.$ every $1-3$ weeks) administration, he experienced 2 disease relapses during the following 3 years. At age 13 switching to adalimumab ( $40 \mathrm{mg}$ every 2 weeks) was decided after additional bowel and joint relapse. At age 14, further relapse occurred: moderate/severe abdominal pain, 2-5 liquid stools (with mucus) per day, perineal pain due to anal fissure, pain in the left foot with limitation of range of motion due to left metatarsal joints arthritis. No oral lesions were noted. Therefore, the patient was admitted, and extensive reassessment was performed (Table 1). Stricture at the ileocecal valve was detected at ileocolonoscopy (Fig. 1A). Histology showed active disease with crypt abscesses (Fig. 1B). Abdomen Magnetic resonance imaging (MRI) showed active disease with increased wall thickness and contrast enhancement in the distal ileum (total length:15-20 mm) with ileal stricture (Fig. 1C). 7-day ciprofloxacin $(2 \mathrm{mg} / \mathrm{Kg} /$ day) and metronidazole $(15 \mathrm{mg} / \mathrm{Kg} /$ day $)$ treatment showed 
Table 1 Baseline clinical and biochemical data

\begin{tabular}{|c|c|c|}
\hline & Result & ReferenceRange \\
\hline Weight (Kg) & 50 & - \\
\hline Weight (Z-score) & -0.46 & $-2-+2$ \\
\hline Height $(\mathrm{cm})$ & 159 & - \\
\hline Height (Z-score) & -1.10 & $-2-+2$ \\
\hline BMI & 20 & - \\
\hline BMI (Z-score) & 0.10 & $-2-+2$ \\
\hline PCDAI & 50 & $<10$ \\
\hline Stool consistency type & 6 & $3-4$ \\
\hline Glucose (mmol/L) & 4.5 & $3.3-6.1$ \\
\hline Lactate $(\mathrm{mmol} / \mathrm{L})$ & 1.8 & $<2.2$ \\
\hline Total cholesterol (mmol/L) & 1.4 & $3.4-5.3$ \\
\hline Triglycerides (mmol/L) & 0.4 & $0.5-1.6$ \\
\hline Uric acid (mmol/L) & 0.26 & $0.13-0.39$ \\
\hline AST (U/L) & 12 & $0-34$ \\
\hline ALT (U/L) & 7 & $0-55$ \\
\hline Albumin (g/L) & 37 & $34-48$ \\
\hline Creatinine (mg/dL) & 0.66 & $0.60-1.10$ \\
\hline Blood Urea Nitrogen (mg/dL) & 13 & $18-45$ \\
\hline eGFR (ml/min/1.73 m2) & 132.6 & 100.9-133.3 \\
\hline White blood cells (WBC)/ $\mu \mathrm{L}$ & 3010 & $5000-15,000$ \\
\hline 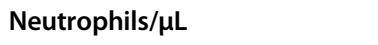 & 1490 & $1300-8500$ \\
\hline 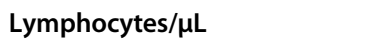 & 1370 & $1300-8500$ \\
\hline Hemoglobin (g/dL) & 8.8 & $11.5-14.0$ \\
\hline Hematocrit (\%) & 33 & $33-35$ \\
\hline Platelets/ $\mu \mathrm{L}$ & 274,000 & $140,000-440,000$ \\
\hline Fibrinogen (mg/dL) & 233 & $160-350$ \\
\hline $1,5 A G(\mu M)$ & 155 & - \\
\hline 1,5AG6P $(\mu \mathrm{M})$ & 1.35 & - \\
\hline $\mathrm{CRP}(\mathrm{mg} / \mathrm{dL})$ & 2.8 & $<0.5$ \\
\hline $\operatorname{ESR}(\mathrm{mm} / \mathrm{h})$ & 35 & $<20$ \\
\hline Adalimumab $(\mu \mathrm{g} / \mathrm{ml})$ & $<0.5$ & $5-10$ \\
\hline Anti-adalimumab lgG (ng/ml) & 62.3 & $<2.5$ \\
\hline 24-h urine protein $(\mathrm{mg} / 24 \mathrm{~h})$ & $<200$ & $<200$ \\
\hline 24-h urine glucose $(\mathrm{mg} / 24 \mathrm{~h})$ & not detected & not detected \\
\hline Fecal calprotectin $(\mu \mathrm{g} / \mathrm{g})$ & 253 & $<100$ \\
\hline
\end{tabular}

PCDAl: Pediatric Crohn's Disease Activity Index; eGFR: estimated glomerular filtration rate; 1,5AG:1,5-anhydroglucitol; 1,5AG6P: 1,5-anhydroglucitol-6phosphate; CRP: C-reactive protein; ESR: erythrocyte sedimentation rate within the first hour

no benefit. Ibuprofen patch was partly effective on arthralgia. Since anti-adalimumab antibodies together with undetectable plasma adalimumab were also detected (Table 1), this treatment was withdrawn (hospital day 5) and ileocecal resection was proposed. $4.8 \mu \mathrm{gG}-\mathrm{CSF} / \mathrm{Kg}$ every other day (i.e., $2.4 \mu \mathrm{g} \mathrm{G}-\mathrm{CSF} / \mathrm{Kg} /$ day) was continued.
Off-label treatment with empagliflozin was also discussed with the patient's family. After oral and written informed consent for this individual treatment, ileocecal resection was postponed, and the patient was started with empagliflozin (day 0 , hospital day 16). The starting dose was $5 \mathrm{mg} /$ day $(0.1 \mathrm{mg} / \mathrm{Kg} /$ day); the dose was further increased to $5 \mathrm{mg}$ twice a day $(0.2 \mathrm{mg} / \mathrm{Kg} /$ day $)$ on day + 3 and $10 \mathrm{mg}$ twice a day $(0.4 \mathrm{mg} / \mathrm{Kg} /$ day $)$ on day +7 . Ciprofloxacin was stopped on day -1 ; metronidazole was withdrawn on day +3 . The dietary regimen was continued as usual (24-h GDF). No significant changes in vital signs and no serious adverse events were observed. On day +22 urinary nitrites (with no leukocytes) were detected, with no associated symptoms. Urine culture was ordered and oral cefixime $(8 \mathrm{mg} / \mathrm{Kg} /$ day $)$ was started (being urinary tract infections common side effects of empagliflozin and considering the risk of metabolic decompensation in case of infection). On day +27 urine culture tested negative and oral cefixime was withdrawn. Subsequent urinalysis tested normal. 24-h urine glucose was absent on day -2 and tested constantly increased after treatment initiation (range 13,212-30,775 $\mathrm{mg} / 24 \mathrm{~h}$ ).

Perineal pain and anal fissure improved after 3 days of treatment and disappeared on day +6 . On day +3 pain in the left foot improved and on day +5 metatarsal swelling was reduced; starting from day +20 no signs or symptoms of arthritis were noted. On day +232 his weight (Z-score: - 0.49), height (Z-score: - 1.03) and BMI (Z-score:0.01) were comparable to baseline. The stool frequency went down to $1-2 \mathrm{x}$ day after 1 week of treatment and $1 \mathrm{x}$ every 2 days after 1 month of treatment. The stool consistency switched from type 6 to type 5 after 2 weeks of treatment and type 4 after 1 month of treatment. The PCDAI decreased from $50($ day -1$)$ to $20($ day +7$)$ to $5($ day +15$)$. Fecal calprotectin increased up to $+40 \%$ during the first month of treatment with subsequent decrease. 7.5 months after starting with empagliflozin its value was $-48 \%$ compared to baseline and almost normalised (Fig. 2A). Similarly, CRP values increased during the first 2 week of treatment and eventually normalised (occasional spikes occurred). ESR values decreased by $34 \%$ after 2 weeks of treatment and normalised after 3 months of treatment. Hemoglobin concentrations constantly increased from the first week of treatment and eventually normalised 5.5 months after starting with empagliflozin (Fig. 2B).

Abdomen MRI performed at +85 days showed $-45 \%$ wall thickness and $-63 \%$ disease length in the distal ileum (total length $5.5 \mathrm{~cm}$ ) together with ileal stricture (Fig. 3A). At +161 days ileocolonoscopy showed unchanged stricture at the ileocecal valve (Fig. 3B); histology showed no signs of active disease (Fig. 3C). At the time no significant change in spleen longitudinal diameter was noted (Z-score: baseline: $+8.75 ;+161$ days: +7.52$)$. 
A

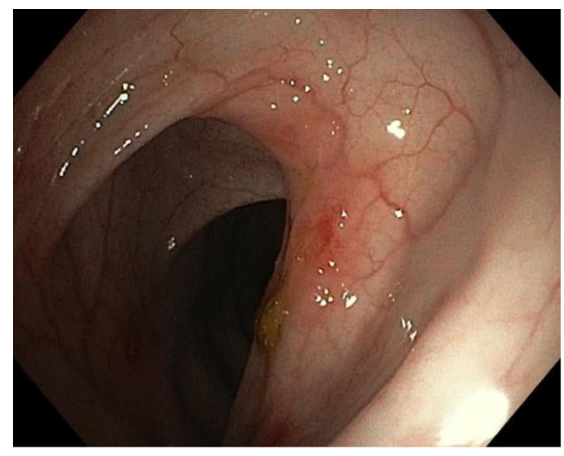

C

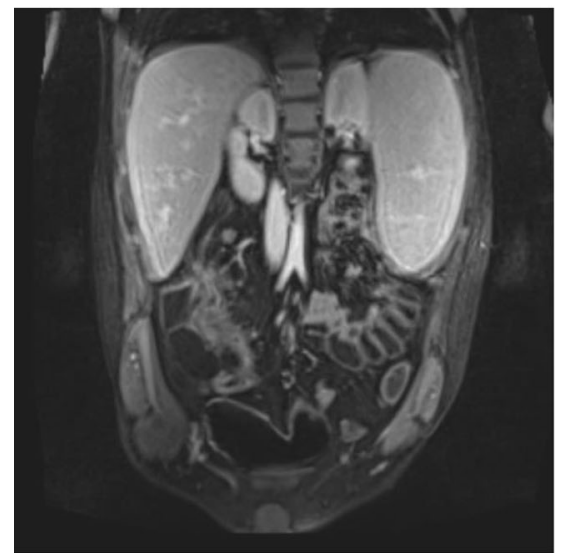

B

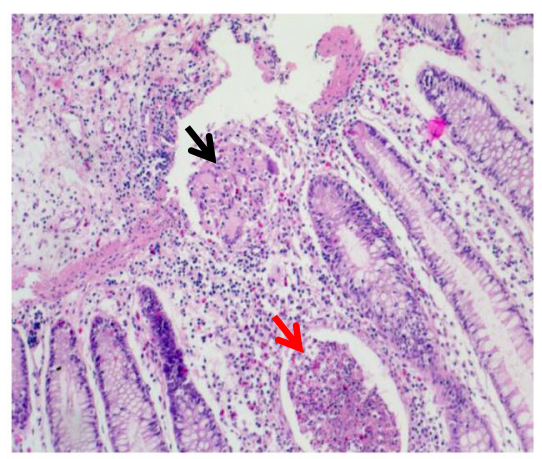

Fig. 1 Bowel morphology at baseline. A lleocolonoscopy: ulcerated and ileocecal valve stricture with impossibility to pass through with the scope (Paris classification A1b, L1, B2, G0; SES-CD: 3). B Histology (colonic mucosa): architectural irregularity and a mild patchy increase of lamina propria cells with neutrophilic and eosinophilic infiltration, crypt abscesses (red arrow) and an epithelioid cell granuloma (black arrow) indicating active disease. C Abdomen MRl: active disease with increased wall thickness (max: $10 \mathrm{~mm}$ ), diffusion restriction and contrast enhancement in the distal ileum (total length:15-20 cm) and ileal stricture; mesenteric hypertrophy (creeping fat) and lymphadenopathy and conglomerated bowel loops (right lower quadrant) are also shown. SES-CD: simplified endoscopic score for Crohn's disease

On day +163 oral refeeding was proposed. Following discussion with the family, the patient was switched from $24-\mathrm{h}$ ( $5 \mathrm{mg}$ carbohydrates $/ \mathrm{Kg} / \mathrm{min}$ ) to $19-\mathrm{h}$ (4 p.m.11 a.m.) (5 mg carbohydrates/Kg/min) GDF. In the "GDF-free" hours one morning snack (11 a.m.) and lunch (01.30 p.m.) were included in the hospital setting, providing an overall carbohydrate intake of $2.2 \mathrm{~g} / \mathrm{Kg}$ (i.e., $7.6 \mathrm{mg}$ carbohydrates $/ \mathrm{Kg} / \mathrm{min}$ ). With such a scheme, the patient's fasting time changed from 0 to $2.5 \mathrm{~h}$ during the "GDF-free" hours. Capillary glucose concentrations (checked every $30 \mathrm{~min}$ on 3 consecutive days during the $5 \mathrm{~h}$ without gastric drip feeding) were $>3.9 \mathrm{mmol} / \mathrm{L}$ (range 4.9-7.2 mmol/L). Neither gastrointestinal symptoms/signs nor changes in stool frequency/features were reported during the subsequent 2-month follow-up; on day +232 fecal calprotectin almost normalised (Fig. 2A).

ANC showed wide variations before empagliflozin $(340-4720 / \mu \mathrm{L})$ with reduced fluctuations $(580-2990 / \mu \mathrm{L})$ after empagliflozin was started. 1,5-AG and 1,5-AG6P concentrations are presented in Additional file 1. The GCSF dose was gradually decreased and finally set to $4.8 \mu \mathrm{g}$ G-CSF/Kg every 3 days (i.e. $1.6 \mu \mathrm{g} \mathrm{G-CSF/Kg/day).}$ Lactate concentrations stayed within the reference range $(1.3-2.3 \mathrm{mmol} / \mathrm{L})$. A slight increase in cholesterol and TG concentrations (which reached the reference range) was observed (Additional file 2A). Uric acid concentrations stayed normal and allopurinol was gradually discontinued (Additional file 2B). Liver and kidney function were regularly checked and tested normal.

Capillary glucose values were below $<3.3 \mathrm{mmol} / \mathrm{L}$ (range $2.6-3.3 \mathrm{mmol} / \mathrm{L}$ ) on $20 / 32$ low-glucose events measured by FGM within the first week of treatment and promptly increased upon glucose administration via the feeding tube. No signs or symptoms of hypoglycemia were reported. Occasional (2-4 times per month) asymptomatic mild hypoglycemias (range $2.7-3.3 \mathrm{mmol} /$ L) occurred during the subsequent 6-month capillary glucose self-monitoring. Data on FGM monitoring are 


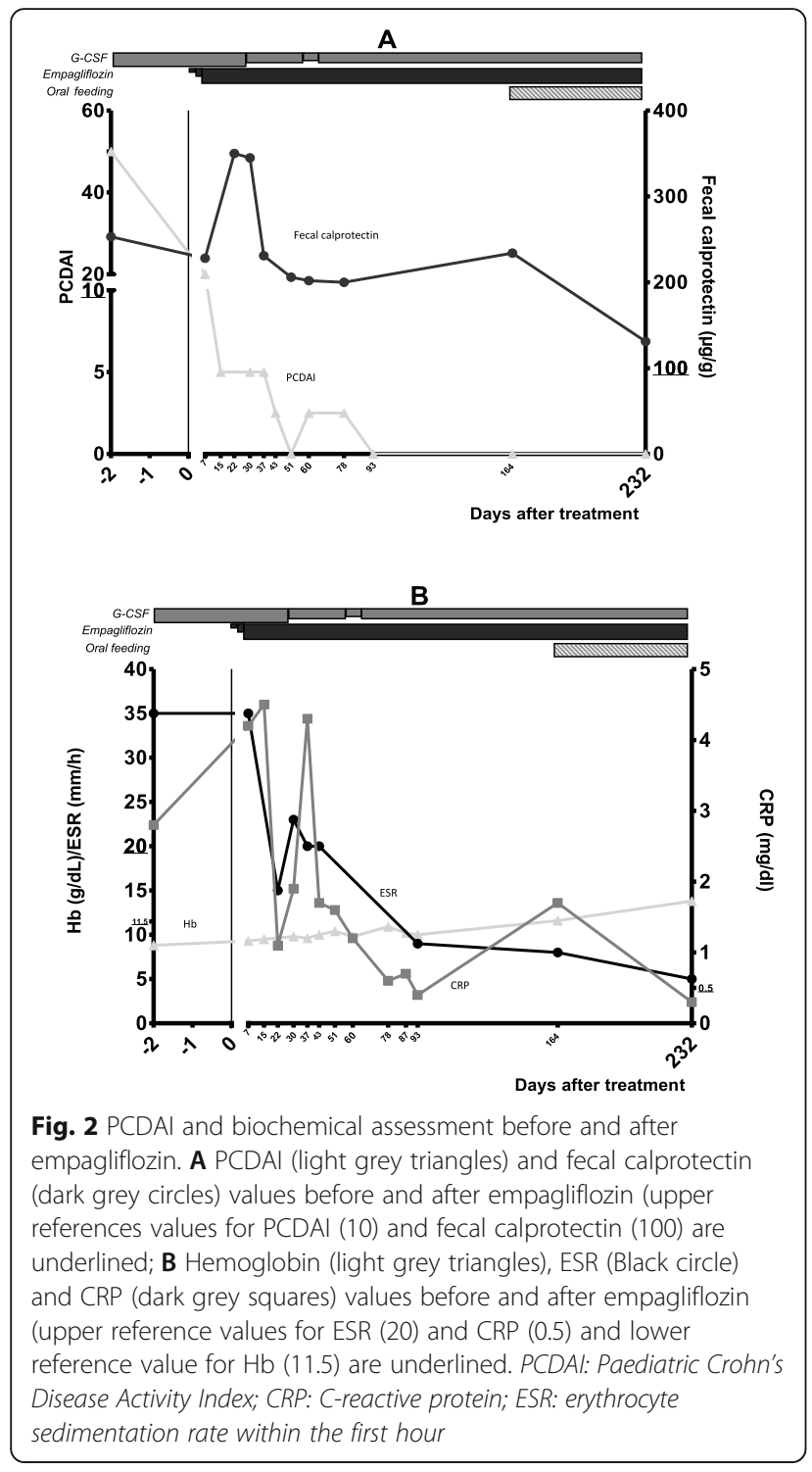

shown in Additional file 3. A substantial decrease in TBR as well as an increase in TIR were observed (also after oral refeeding was started). The patient's QoL score improved from 37.64 (baseline) to $74.44($ day +232$)$.

\section{Discussion and conclusions}

The management of IBD is still challenging in GSD Ib as its pathogenesis remains [1,3]. Conventional treatments (i.e., corticosteroids, immunomodulators, biological agents) are sometimes ineffective and/or associated with side effects (e.g. leucopenia, anemia, diarrhea) and patients might eventually need surgery [18]. Being the cornerstone of treatment for neutropenia, G-CSF has also led to IBD remission in some GSD Ib patients [19]. However, its efficacy is variable and long-term G-CSF administration may cause side effects (e.g. enlarged spleen) and increased risk for malignancies (e.g. acute myeloid leukemia, myelodysplastic syndrome)
$[20,21]$. Therefore, more effective treatments are required to improve patients prognosis and quality of life.

Although previous reports have shown possible benefit of empagliflozin on gastrointestinal symptoms [12, 13], follow-up data on bowel (macro/microscopic) morphology are not available. In the case herein reported, we presented comprehensive gastrointestinal assessment in a child with GSD Ib, showing clear benefit of empagliflozin administration on Chron disease-like enterocolitis. No symptomatic hypoglycemias and no adverse events were associated with empagliflozin administration.

Clinical improvement was noted within the first week of treatment and eventually led to normal stool frequency/consistency and PCDAI normalisation. Clinical remission occurred within the first month of treatment. Biochemical improvement occurred within the first 2 weeks of treatment and remission was documented after 2 months of treatment. Strikingly, the benefit on clinical picture and hemoglobin concentrations appeared as soon as the end of the first week. Morphology studies also showed partial IBD remission within 3-6 months of treatment. Notably, a dramatic improvement in the disease length and activity was documented on the abdomen MRI after 3 months of treatment as well as histologic remission after 5.5 months of treatment. Empagliflozin allowed to postpone ileocecal resection (and possibly decrease the length of the bowel segment to be resected) in the present case. However, no major endoscopic changes were noted 5.5 months after starting treatment. Those data show that empagliflozin may be effective in healing the inflammatory lesions/strictures but might not be able to reverse fibrotic strictures once established. Such conclusion suggests early empagliflozin administration in GSD Ib patients with IBD before the onset of (irreversible) intestinal fibrosis. $0.4 \mathrm{mg} / \mathrm{Kg} /$ day empagliflozin were administered in the present case. Previous study showed $0.3-0.7 \mathrm{mg} / \mathrm{Kg} /$ day to lay within the therapeutic window for neutropenia [12]. It is still unclear if higher doses can be more effective or whether a specific dose range might exert special benefit on other disease complications (e.g., IBD, arthritis). Future studies should address this issue.

Besides the effect on IBD, decreased 1,5AG and 1, 5AG6P concentrations as well as higher/more stable neutrophil count were also documented after starting with empagliflozin. Based on those data, the G-CSF dose was decreased by $33 \%$ in the present case. Undoubtedly, reducing G-CSF dose can decrease the risk of side effects and malignancies associated with its long-term administration. However, not all GSD Ib patients treated with empagliflozin are able to discontinue G-CSF [12]. The reason for such discrepancy is still unclear. Possible role of additional factors contributing to empagliflozin response (e.g., genotype, renal function, glycosylation 
A

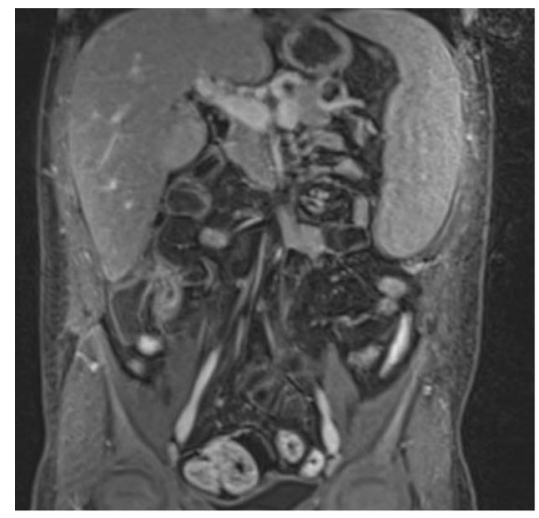

B

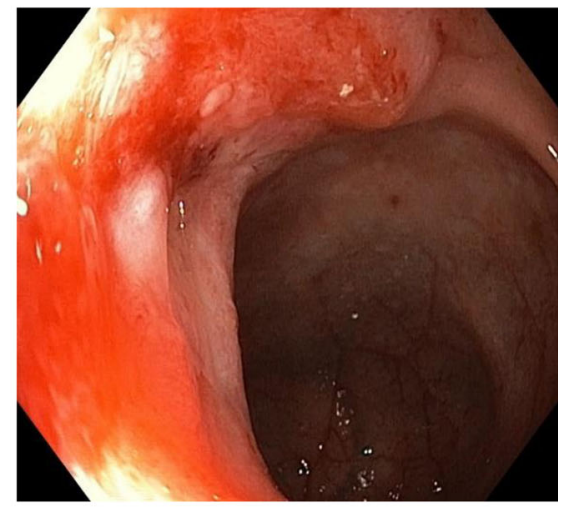

D

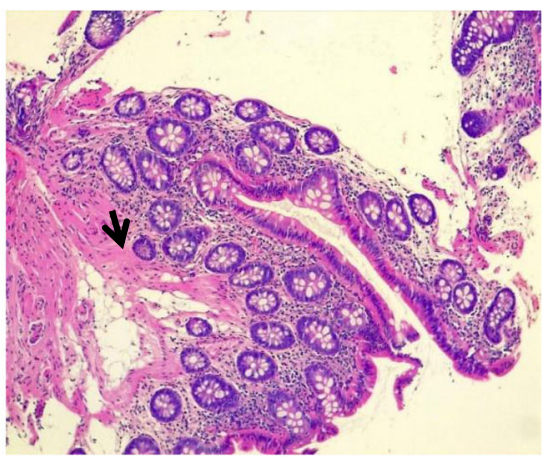

Fig. 3 Bowel morphology after empagliflozin treatment. A MRI (day + 85): decreased wall thickness (max $6.5 \mathrm{~mm})$, decreased diffusion restriction, decreased contrast enhancement in the distal ileum (total length: $5.5 \mathrm{~cm}$ ) together with ileal stricture; stable mesenteric hypertrophy (creeping fat) and lymphadenopathy with no evidence of conglomerated bowel loops (right lower quadrant) are also shown. B-C. lleocolonoscopy (day + 161): ileocecal valve ulcer and stricture with the impossibility to pass through with the scope (Paris classification A1b, L1, B2, G0; SES-CD: 3). D Histology (day +161 , colonic mucosa): minimal architectural distortion, increase of lamina propria, associated with muscularis mucosae hypertrophy (black arrow) and adequate gland representation indicating chronic mild colitis with histologic remission. SES-CD: simplified endoscopic score for Crohn's disease

status) should be further addressed for optimal patient selection. The results herein reported also support possible role of disrupted immune response in the pathogenesis of IBD in GSD Ib. Indeed, a role for 1,5AG and $1,5 \mathrm{AG} 6 \mathrm{P}$ in modulating other peripheral blood mononuclear cells has been postulated [11].

Despite 33\% reduction in G-CSF dose, no change in spleen size was observed in the current patient. In 3 out of the 5 previous GSD Ib patients that have been previously described to be treated with empagliflozin and who presented with splenomegaly, 2 showed decreased spleen size only 9 months after starting empagliflozin (G-CSF was discontinued or decreased by $81 \%$, respectively). Despite G-CSF discontinuation, 1 patient showed stable spleen size 3 months after starting empagliflozin
[12]. Longer follow-up studies are warranted to clarify when to expect benefit on splenomegaly.

Additionally, improved metabolic control was noted in the present case. Increase of (low) cholesterol and TG levels and reversal of hyperuricemia (leading to allopurinol discontinuation) were detected after empagliflozin administration. Likely, normalisation of plasma cholesterol and TG were secondary to intestinal healing in the present case. FGM data showed stable glucose levels and eventually no hypoglycemia was detected. Strikingly, the patient also experienced (limited) amount of time in the "above-range" (Additional file 3). Such findings are in line with previous report [12] suggesting that IBD might concur to metabolic control in GSD Ib patients by limiting intestinal glucose absorption. Interestingly, recent 
research has shown the impact of life-long diet on gut microbiota in GSD Ib [22]. FGM may constitute an additional, minimally invasive monitoring tool for GSD Ib patients.

Not only led empagliflozin to improved clinical conditions and biochemical/morphological markers but also allowed drug dose reduction/discontinuation in the present case. As a matter of fact, the patient was switched from 4-drug (i.e., adalimumab, allopurinol, G-CSF, ibuprofen patch) to 2-drug (G-CSF, empagliflozin) regimen with a simplified drug schedule. Notably, the number of painful G-CSF injections was decreased. Benefits on healthcare costs (empagliflozin is less expensive than G-CSF and biologic therapies for IBD) as well as reduced healthcare use by GSD Ib patients can also be expected. Indeed, the gross monthly financial burden for medication decreased by $59 \%$ in the present case (3586 € vs $1467 €)$.

Improved QoL was also observed after empagliflozin administration. Patients with IBD show increased prevalence of psychological disturbances like depression and anxiety [23]. In the present case, the patient agreed on restarting oral feeding after 8 years, allowing (in part) weaning from tube feeding. This result suggests that, by improving the IBD-related symptoms, empagliflozin can also exert a positive effect on psychosocial health and well-being in patients with GSD Ib.

Although renal function was constantly normal in the present case, future studies should assess the effect of empagliflozin on renal function in GSD Ib patients [24, 25].

Overall, the present case shows that empagliflozin administration is safe and effective in inducing IBD remission in GSD Ib patients and can postpone surgery. It also improves neutrophil count and metabolic control. Since this is the first case documenting comprehensive longitudinal IBD morphology follow-up in a patient with GSD Ib treated with empagliflozin, future studies are needed to confirm its safety and efficacy over time and assess its benefit in various disease stages. As empagliflozin has the potential to change the natural history and management of GSD Ib patients, the development of an international collaborating networks for systematic data collection on its safety and efficacy is worthy.

\section{Abbreviations}

ANC: Absolute neutrophil count; CRP: C-reactive protein; ESR: Erythrocyte sedimentation rate; FGM: Flash glucose monitoring; GSD Ib: Glycogen storage disease type Ib; IBD: Inflammatory bowel disease; PCDAI: Pediatric Crohn disease activity index; QoL: Quality of life; TG: Triglycerides; 1,5AG: 1,5anhydroglucitol; 1,5AG6P: 1,5-anhydroglucitol-6-phosphate

\section{Supplementary Information}

The online version contains supplementary material available at https://doi. org/10.1186/s13052-021-01100-w.

Additional file 1. Neutrophil count (light grey triangles), 1,5AG (black circles) and 1,5AG6P (dark grey squares) before and after empagliflozin.
Plasma 1,5-AG concentration dropped from $\pm 250 \mu \mathrm{M}$ before treatment to $\pm 50 \mu \mathrm{M}$ after 2 weeks on empagliflozin. Concentration of 1,5-AG stayed relatively constant until day +164 , before a change in the diet introducing a daily oral intake of carbohydrates. On day +232 , approximately 2 months after this change, plasma 1,5-AG was only very slightly increased to $60 \mu \mathrm{M}$. After treatment, 1,5-AG6P present in leukocytes and measured in whole blood samples was reduced by 4- to 5-fold when compared to values before starting empagliflozin. 1,5AG: 1,5anhydroglucitol; 1,5AG6P: 1,5-anhydroglucitol-6-phosphate.

Additional file 2. (A) Plasma cholesterol (grey triangles) and TG (black circles) before and after empagliflozin (reference values for cholesterol (3-5) and TG (0.5-1.5) are underlined); (B) Plasma uric acid concentrations before and after empagliflozin (reference values are underlined). TG: triglycerides.

Additional file 3. Flash glucose monitoring data.

\section{Acknowledgements}

The authors would like to thank Rosaria Tuzzi and Carmen Rosano for their support with the collection of samples.

\section{Authors' contributions}

$A R, E M, S F, M M, C M, M D A, E M$ were involved in data collection, analysed and interpreted the patient data and wrote the first draft of the manuscript. MVDC, PS, TGJD, AS, GP were involved in data collection, analysed and interpreted the patient data and critically revised the manuscript. All the authors made substantial contributions to the conception or design of the work. All authors read and approved the final manuscript.

\section{Funding}

This work was supported by a research funding donation from Vitaflo Italia to AR and GP (no award/grant number) and the 01/2020 scholarship from Associazione Italiana Glicogenosi to AR and TGJD.

\section{Availability of data and materials}

The datasets used and/or analysed during the current study are available from the corresponding author on reasonable request.

\section{Declarations}

Ethics approval and consent to participate

Written informed consent was obtained from the patient's parents. A copy of the written consent is available for being reviewed by the Editor-in-Chief of this journal.

\section{Consent for publication}

Written informed consent was obtained from the patient's parents. A copy of the written consent is available for being reviewed by the Editor-in-Chief of this journal.

\section{Competing interests}

The authors declare that there is no potential conflict of interest related to this work. There are no prior publications or submissions with any overlapping information, including studies and patients.

\section{Author details}

${ }^{1}$ Department of Translational Medical Sciences, Section of Pediatrics, University of Naples "Federico II", Naples, Italy. 2Section of Metabolic Diseases, Beatrix Children's Hospital, University Medical Center Groningen, University of Groningen, P.O. Box 30.001, 9700 RB Groningen, The Netherlands. ${ }^{3}$ Groupe de Recherches Metaboliques, de Duve Institute, UC Louvain (Université Catholique de Louvain), B-1200 Brussels, Belgium. ${ }^{4}$ Section of Medical Imaging, Department of Advanced Biomedical Sciences, University of Naples Federico II, Naples, Italy. ${ }^{5}$ Section of Pathology, Department of Advanced Biomedical Sciences, University of Naples Federico II, Naples, Italy. ${ }^{6}$ Telethon Institute of Genetics and Medicine, Pozzuoli, Italy. 
Received: 16 April 2021 Accepted: 3 June 2021

Published online: 02 July 2021

\section{References}

1. Kishnani PS, Austin SL, Abdenur JE, et al. Diagnosis and management of glycogen storage disease type I: a practice guideline of the American College of Medical Genetics and Genomics. Genet Med. 2014;128:1-29.

2. Rake JP, Visser G, Labrune P, Leonard JV, Ullrich K, Smit. Guidelines for management of glycogen storage disease type I - European study on glycogen storage disease type I (ESGSD I). Eur J Pediatr. 2002;161(1):S112-9. https://doi.org/10.1007/BF02680007.

3. Visser G, Rake J, Labrune P, et al. Consensus guidelines for management of glycogen storage disease type $1 \mathrm{~b}$ - European study on glycogen storage disease type 1. Eur J Pediatr. 2003;161:S120-3.

4. Visser G, Rake JP, Fernandes J, Labrune P, Leonard JV, Moses S, et al. Neutropenia, neutrophil dysfunction, and inflammatory bowel disease in glycogen storage disease type lb: results of the European study on glycogen storage disease type I. J Pediatr. 2000;137(2):187-91. https://doi. org/10.1067/mpd.2000.105232.

5. Melis D, Pivonello R, Parenti G, Della Casa R, Salerno M, Lombardi G, et al. Increased prevalence of thyroid autoimmunity and hypothyroidism in patients with glycogen storage disease type I. J Pediatr. 2007;150(3):300-5 305.e1.

6. Melis D, Della Casa R, Balivo F, Minopoli G, Rossi A, Salerno M, et al. Involvement of endocrine system in a patient affected by glycogen storage disease 1b: speculation on the role of autoimmunity. Ital J Pediatr. 2014; 40(1):30. https://doi.org/10.1186/1824-7288-40-30.

7. Jun HS, Weinstein DA, Lee YM, Mansfield BC, Chou JY. Molecular mechanisms of neutrophil dysfunction in glycogen storage disease type Ib. Blood. 2014;123(18):2843-53. https://doi.org/10.1182/blood-2013-05-502435.

8. Melis D, Carbone F, Minopoli G, La Rocca C, Perna F, De Rosa V, et al. Cutting edge: increased autoimmunity risk in glycogen storage disease type $1 \mathrm{~b}$ is associated with a reduced engagement of glycolysis in T cells and an impaired regulatory T cell function. J Immunol. 2017;198(10):3803-8. https:// doi.org/10.4049/jimmunol.1601946.

9. Rossi A, Simeoli C, Salerno M, Ferrigno R, Della Casa R, Colao A, et al. Imbalanced cortisol concentrations in glycogen storage disease type I: evidence for a possible link between endocrine regulation and metabolic derangement. Orphanet J Rare Dis. 2020;15(1):99. https://doi.org/10.1186/ s13023-020-01377-w.

10. Peeks F, Boonstra W, de Baere $L$, et al. Research priorities for liver glycogen storage disease: an international priority setting partnership with the James Lind Alliance. J Inherit Metab Dis. 2020;43(2):279-89. https://doi.org/10.1002/ jimd.12178.

11. Veiga-da-Cunha M, Chevalier N, Stephenne X, Defour JP, Paczia N, Ferster A, et al. Failure to eliminate a phosphorylated glucose analog leads to neutropenia in patients with G6PT and G6PC3 deficiency. Proc Natl Acad Sci U S A. 2019;116(4):1241-50. https://doi.org/10.1073/pnas.1816143116.

12. Wortmann SB, Van Hove JLK, Derks TGJ, Chevalier N, Knight V, Koller A, et al. Treating neutropenia and neutrophil dysfunction in glycogen storage disease IB with an SGLT2-inhibitor. Blood. 2020;136(9):1033-43. https://doi. org/10.1182/blood.2019004465.

13. Grünert SC, Elling R, Maag B, Wortmann SB, Derks TGJ, Hannibal L, et al. Improved inflammatory bowel disease, wound healing and normal oxidative burst under treatment with empagliflozin in glycogen storage disease type Ib. Orphanet J Rare Dis. 2020;15(1):218. https://doi.org/10.1186/ s13023-020-01503-8.

14. Al-Jobori H, Daniele G, Cersosimo E, et al. Empagliflozin and kinetics of renal glucose transport in healthy individuals and individuals with type 2 diabetes. Diabetes. 2017;66(7):1999-2006. https://doi.org/10.2337/db17-0100.

15. Hyams JS, Ferry GD, Mandel FS, Gryboski JD, Kibort PM, Kirschner BS, et al. Development and validation of a pediatric Crohn's disease activity index. J Pediatr Gastroenterol Nutr. 1991;12(4):439-47.

16. Danne, et al. International Consensus on Use of Continuous Glucose Monitoring. Diabetes Care. 2017;40(12):1631-40. https://doi.org/10.2337/dc1 7-1600.

17. Sechi A, Deroma L, Paci S, Lapolla A, Carubbi F, Burlina A, et al. Quality of life in adult patients with glycogen storage disease type I: results of a multicenter Italian study. JIMD Rep. 2013. https://doi.org/10.1007/8904_2 013_283.
18. Yamaguchi T, Ihara K, Matsumoto T, Tsutsumi Y, Nomura A, Ohga S, et al. Inflammatory bowel disease-like colitis in glycogen storage disease type $1 \mathrm{~b}$ Inflamm Bowel Dis. 2001;7(2):128-32. https://doi.org/10.1097/00054725-2001 05000-00008.

19. Alsultan A, Sokol RJ, Lovell MA, Thurman G, Ambruso DR. Long term G-CSFinduced remission of ulcerative colitis-like inflammatory bowel disease in a patient with glycogen storage disease $\mathrm{lb}$ and evaluation of associated neutrophil function. Pediatr Blood Cancer. 2010;55(7):1410-3. https://doi. org/10.1002/pbc.22706.

20. Li AM, Thyagu S, Maze D, Schreiber R, Sirrs S, Stockler-Ipsiroglu S, et al. Prolonged granulocyte colony stimulating factor use in glycogen storage disease type $1 \mathrm{~b}$ associated with acute myeloid leukemia and with shortened telomere length. Pediatr Hematol Oncol. 2018;35(1):45-51.

21. Khalaf D, Bell H, Dale D, Gupta V, Faghfoury H, Morel CF, et al. A case of secondary acute myeloid leukemia on a background of glycogen storage disease with chronic neutropenia treated with granulocyte colony stimulating factor. JIMD Rep. 2019;49(1):37-42.

22. Ceccarani C, Bassanini G, Montanari C, Casiraghi MC, Ottaviano E, Morace G, et al. Proteobacteria overgrowth and butyrate-producing taxa depletion in the gut microbiota of glycogen storage disease type 1 patients. Metabolites. 2020;10(4):133. https://doi.org/10.3390/metabo10040133.

23. Neuendorf R, Harding A, Stello N, Hanes D, Wahbeh H. Depression and anxiety in patients with inflammatory bowel disease: A systematic review Psychosom res. Aug. 2016;87:70-80

24. Melis D, Cozzolino M, Minopoli G, Balivo F, Parini R, Rigoldi M, et al. Progression of renal damage in glycogen storage disease type I is associated to hyperlipidemia: a multicenter prospective Italian study. J Pediatr. 2015;166(4):1079-82.

25. Wanner C, Inzucchi SE, Lachin JM, Fitchett D, von Eynatten M, Mattheus M, et al. Empagliflozin and Progression of Kidney Disease in Type 2 Diabetes. N Engl J Med. 2016;375(18):1801-2.

\section{Publisher's Note}

Springer Nature remains neutral with regard to jurisdictional claims in published maps and institutional affiliations.
Ready to submit your research? Choose BMC and benefit from:

- fast, convenient online submission

- thorough peer review by experienced researchers in your field

- rapid publication on acceptance

- support for research data, including large and complex data types

- gold Open Access which fosters wider collaboration and increased citations

- maximum visibility for your research: over $100 \mathrm{M}$ website views per year

At BMC, research is always in progress.

Learn more biomedcentral.com/submissions 